\title{
UNA INTRODUCCIÓN A LA LITERATURA CIENTÍFICA
}

\author{
GARCIA MOLINA, R. ${ }^{1}$ y ABRIL, I. $^{2}$ \\ ${ }^{1}$ Departamento de Física. Universidad de Murcia. Apartado 4021. 30080 Murcia. \\ 2 Departament de Física Aplicada. Universitat d'Alacant. Apartat 99. 03080 Alacant.
}

\begin{abstract}
SUMMARY
We discuss the convenience of an early introduction of undergraduate students to the reading of scientific papers, mostly divulgative or historical. This can encourage them to deeper readings and to search and discover in the scientific writings new sources of learning and pleasure. The aim of this activity is twofold: mainly, to approach the students to bibliographical sources different from the traditional textbooks and, collaterally, to habituate them to get acquainted with scientific information in foreign languages (mostly English). In this manner, the students can get further knowlegde and they can also learn new points of view or different topics from those commonly discussed during a typical academic course.
\end{abstract}

\section{INTRODUCCIÓN}

«El castellano es apropiado para hablar con dios, el francés con los amigos, el aiemán con los enemigos y el italiano con las mujeres». La frase anterior, atribuida al emperador CarIos Ide España (y V de Alemania), admite diversos comentarios y se puede estar de acuerdo o discrepar en parte o totalmente. Pero sin lugar a dudas, en la actualidad podríamos añadir que el inglés es el lenguaje empleado para comunicarse en ciencia. La inmensa mayoría de publicaciones y de reuniones cientéficas tienen el inglés como lengua principal, cuando no única (Gibbs, 1995).

La educación secundaria (como tal nos referimos a la educación previa a la universitaria) contempla Ia enseñanza de una lengua extranjera que, por lo general, es el inglés, y, en menor medida, el francés. Sin embargo, aunque los estudiantes adquieren unos conocimientos básicos del idioma extranjero estudiado, raras veces (por no decir nunca) los aplican cuando comienzan a estudiar alguna disciplina científica. La explicación de esto resi- de en que las asignaturas cursadas en los primeros años de las carreras científicas o técnicas disponen normalmente de una bibliografía abundante en castellano. Esto se debe a que, aparte de los textos escritos por autores castellanopariantes, las principales editoriales científicas traducen un buen número de libros de texto de entre los que gozan de reconocido prestigio en el mundo anglosajón. La inversión económica que supone la puesta en el mercado de estas traducciones está compensada por el amplio mercado castellanoparlante (España y América Latina principalmente). En cambio, en otros países con mucha menos población potencialmente consumidora de textos científicos que la de habla castellana (como es el caso de los países escandinavos, por ejemplo), los estudiantes universitarios manejan desde los primeros cursos bibliografía en inglés. Puesto que en su momento padecimos en carne propia las consecuencias de no desenvolvernos con soltura en el inglés científico (que es más accesible que el literario o el coloquial), decidimos desarrollar entre nuestros alumnos del pri- 
mer curso de las licenciaturas en química y en biología la experiencia de invitarlos a conocer literatura científica en inglés.

Pero lo que en un principio estaba pensado como una propuesta de trabajo para alertar a los estudiantes sobre la conveniencia de desenvolverse con soltura en la lengua mayoritaria en la ciencia, nos llevó a dotar a esta actividad de mayor contenido, aprovechándola para que, al mismo tiempo, tomaran contacto con el mundo de las publicaciones científicas periódicas. De hecho, se invirtió la prioridad de intereses, pues el principal objetivo se convirtió en acercar a los estudiantes a las fuentes de donde emana, y a través de las cuales se intercambia la información científica, $y$, colateralmente, los estudiantes podrían constatar, desde bien temprano en su formación académica, el uso mayoritario de la lengua inglesa en la comunicacion cientiffica. Claro está que el tipo de lecturas asequibles a un estudiante de primer curso universitario se encuentra en publicaciones de carácter divulgativoy, en contadas ocasiones, en algunas revistas de contenidos más avanzados (pero en este caso, seleccionando muy cuidadosamente el material de lectura).

\section{FORMA Y CONTENIDO}

Por forma nos referimos al modo en que se presenta la información escrita que se transmite a los estudiantes de las licenciaturas antes mencionadas: la lengua utilizada, así como el medio empleado.

En el ámbito universitario del Estado español, la lengua es la castellana, incluso en las comunidades autónomas donde coexiste otra lengua oficial, pues a nivel universitario todavía no se dispone de abundante bibliografía científica en catalán, vasco, etc. -excepto algunos títulos aislados-; aunque cada vez son más los profesionales comprometidos en la elaboración de material didáctico a nivel universitario escrito en las lenguas antes citadas. El medio material mediante el cual se presentan y transmiten los conocimientos a los estudiantes es, por lo general, el libro de texto. Pero lo cierto es que, si bien no hay ningún inconveniente en seguir los cursos cuya bibliograf́a esté en castellano, cuando se llega a los últimos años de la licenciatura o cuando se finaliza la misma, es necesario manejar bibliografía en alguna lengua extranjera (principalmente inglés), sobre todo si los estudiantes desean proseguir su actividad en el mundo de Ia investigación. Además, mucha de la información que ha de manejarse no se encontrará en los libros de texto, con cuyo uso suelen estar familiarizados los estudiantes, sino en textos más específicos, como son las revistas especializadas, las memorias de los laboratorios de investigacion, etc.

Por contenido entendemos el tipo de mensaje que les llega a los estudiantes cuando leen un libro de texto convencional. Los textos que manejan los alumnos contienen los resultados estables de revoluciones científicas pasadas (Kuhn, 1975) y únicamente suministran las respuestas dadas por la ciencia, sin discutir las preguntas que fueron el origen de tales respuestas (Otero, 1989). En general, los estudiantes piensan en la ciencia en términos de solidez y permanencia, y suelen sentirse sorprendidos y desorientados por la imagen de movilidad escurridiza de la investigación de cada día. Por eso, en muchas ocasiones no sólo conviene saber ciencia, sino también cómo se hizo esta ciencia, conociendo el devenir histórico de algunas teorías, conceptos, experimentos, etc. Por ello sería interesante que los estudiantes pudieran acceder a conocimientos cientfficos que todavía no están presentados en la forma tradicional del libro de texto.

Para evitar que les resulte traumático el tener que enfrentarse con esos otros textos al finalizar la carrera, es conveniente hacer conocer a los estudiantes la posibilidad de que en un futuro (no muy lejano) necesitan manejar información que, tanto por su contenido como por su forma, no se encuentra en los textos tradicionales a los cuales están acostumbrados. Para ello nada mejor que proponerles, ya desde los primeros cursos universitarios, la lectura de algunos artículos seleccionados de forma que les resulten interesentes, gratificantes y de no excesiva dificultad (tanto léxica como de contenido científico). Con ello, además de manejar fuentes alternativas a las tradicionales, este tipo de lecturas también cumpliría un objetivo de formación de los alumnos, para que perciban la ciencia no como una sucesión cronologica de verdades absolutas, sino que mediante estas lecturas se les podría inculcar el estudio de la génesis de algunas ideas cientifícas (Rosmorduc, 1993; Lahore, 1993). Así, tendrían una perspectiva de la mentalidad de cada época, que suele estar ligada a la cultura dominante, y condiciona (Kuhn, 1975) el desarrollo de unas líneas determinadas de investigación y no otras'. También podemos aprovechar estas lecturas para contrarrestar las valoraciones negativas (preconcebidas o consecuencia de una experiencia frustrante) que de algunas disciplinas científicas, en especial de la física, tienen los estudiantes (Beall, 1993). Por último, otro objetivo que podría alcanzarse es que los estudiantes entren en contacto con puntos de vista diferentes o complementarios a los que se les presentan habitualmente en los libros de textos más usuales.

\section{PROPUESTA DE REALIZACIÓN DE LA ACTIVIDAD}

Desde hace varios años hemos ofrecido, a aquellos alumnos que cursan la asignatura de física en el primer ciclo de algunas licenciaturas de ciencias, la posibilidad de leer artículos de temas científicos de divulgación que, además, estaban escritos en inglés ${ }^{2}$. Seguidamente describimos la forma en la que se ha Ilevado a la práctica.

En una fecha dada se le entrega a cada alumno una hoja que contiene resumidamente los objetivos y el procedimiento a seguir para realizar la actividad propuesta, así como una lista de los artículos sugeridos para leer. 
A continuación presentamos dicho resumen:

- Los objetivos son acostumbrarse a manejar fuentes bibliográficas diferentes de los líbros de textos tradicionales, así como a recibir y manipular información científica en lenguas extranjeras, principalmente en inglés. También se pretende ampliar conocimientos, presentar nuevos aspectos y abordar temas diferentes de los tratados habitualmente durante el programa de la asignatura de física de primer curso.

- La actividad propuesta se desarrollará individualmente o en grupos de dos personas. Cada grupo elige un único artículo de entre una lista propuesta. Si desea proponer un tema no contemplado en dicha lista conviene que se lo comunique al profesor con suficiente antelación para que éste pueda encontrar la bibliografía adecuada (si no se encontrase dicha bibliografía habría que volver a elegir el artículo de entre la lista propuesta originalmente).

- Transcurrido un plazo de tiempo razonable, deberá entregarse el trabajo, que constara (aparte de la identificación del grupo y de la lectura elegida) de un resumen muy breve $y$, a ser posible, de conclusiones y de uná valoración personal de la lectura; de hecho, parafraseando a Baierlein (1987), podemos decir que cualquier forma de comentario respetable intelectualmente es válido como resumen o crítica del texto leído.
Conviene que el profesor proponga cuanto antes a los alumnos la actividad a realizar. En concreto, en el caso de asignaturas cuatrimestrales, esto puede hacerse a las pocas semanas de comenzar el curso. Así los estudiantes pueden entregar el trabajo a la vuelta de las vacaciones de Navidad o de Semana Santa, dependiendo de si la asignatura se imparte en el primer o en el segundo cuatrimestre, respectivamente. En todo caso, conviene que los estudiantes dispongan de un plazo de tiempo más que suficiente para poder leer sin agobios un texto (no muy extenso) en inglés. A título orientativo, en la tabla I adjunta presentamos las referencias de algunos de los artículos que más han solicitado los estudiantes de química y de biología (señalados con una $Q$ o una $B$, respectivamente) durante Ios años que hemos llevado a cabo la experiencia ${ }^{3}$.

Como puede observarse, algunos artículos suscitan el interés común de los estudiantes de química y de biología; por ejemplo, aquéllos relacionados con la conservación del medio ambiente. Por otra parte, también se aprecia como otros estudiantes se decantan por temas de física más relacionados con sus respectivos estudios de licenciatura.

Es importante resaltar la conveniencia de seleccionar muy cuidadosamante las lecturas recomendadas, para evitar que resulte frustrante este primer encuentro con textos científicos no tradicionales, pues la percepción

Tabla I

\begin{tabular}{|l|c|}
\hline $\begin{array}{l}\text { «Misconceptions about Eirstein. His works and his views», Journal of Chemical } \\
\text { Education, S7 (1980), pp. 854-862. }\end{array}$ & $\mathrm{Q}$ \\
\hline «The planet of the little prince», Physics Education, 23 (1988), pp. 224-225. & $\mathrm{Q}$ \\
\hline «The physicist as mad scientist», Physics Today (june 1988), pp. 28-37. & $\mathrm{Q}$ \\
\hline $\begin{array}{l}\text { «The persistent stereotype: children's images of scientist», Physics Education, 25 } \\
\text { (1990), pp. I02-105. }\end{array}$ & $\mathrm{Q}$ \\
\hline $\begin{array}{l}\text { «The greenhouse effect: fact or fiction?», CSTU Environmental Information Paper } \\
\text { (1992), p. 4. }\end{array}$ & $\mathrm{Q} \mathrm{B}$ \\
\hline $\begin{array}{l}\text { «The year after. Les cffets biologiques d'une guerre nucléaire", La Recherche, 154 } \\
\text { (Avril 1984), pp. 542-546. }\end{array}$ & $\mathrm{Q} \mathrm{B}$ \\
\hline «Cartoons in science», Physics Education, 26 (1991), pp. 110-114. & $\mathrm{Q} \mathrm{B}$ \\
\hline \begin{tabular}{l} 
«Photobiology of the skin», Physics Bulletin, 35 (1984), pp. 69-72. \\
\hline \begin{tabular}{l} 
«The physics of marine biology», The Physics Teacher, 30 (1992), pp. 554-557. \\
\hline «The physics of medical imaging», Physics Today (July 1983), pp. 36-42.
\end{tabular}
\end{tabular} \\
\hline
\end{tabular}


